\title{
Elevated ozone levels affect metabolites and related biosynthetic genes in tartary buckwheat
}

\author{
Jin Jeon ${ }^{1}$, Seung A Baek ${ }^{2}$, Nam Su Kim ${ }^{1}$, Ramaraj Sathasivam¹, Jong Seok Park ${ }^{1}$, Jae \\ Kwang Kim², and Sang Un Park ${ }^{1}$ \\ ${ }^{1}$ Chungnam National University \\ ${ }^{2}$ Incheon National University
}

April 28, 2020

\begin{abstract}
Global climate change and the industrial revolution have increased the concentration of tropospheric ozone, a photochemical air pollutant that can negatively affect plant growth and crop production. In the present study, we investigated the effects of O3 on the metabolites and transcriptome of tartary buckwheat. A total of 37 metabolites were identified by gas chromatography coupled with time-of-flight mass spectrometry, and principal component analysis was performed to verify the metabolic differences between non-treated and O3-treated Tartary buckwheat. The levels of most metabolites (threonic acid, tryptophan, sucrose, and raffinose) decreased significantly after the O3 treatment. On the contrary, the levels of two anthocyanins, cyanidin 3-O-glucoside and cyanidin 3-O-rutinoside, increased more than 11.36- and 11.43-fold, respectively after the O3 treatment. To assess the effect of $\mathrm{O} 3$ on the genomic level, we analyzed the expression of anthocyanin biosynthesis pathway genes in O3-treated and non-treated buckwheat using quantitative real-time reverse transcription PCR. We found that the expression of all anthocyanin pathway genes increased significantly in the O3-treated buckwheat compared to that in the non-treated buckwheat. Altogether, our results suggested that $\mathrm{O} 3$ affected the transcripts and metabolites of tartary buckwheat, which would eventually cause phenotypic changes in plants.
\end{abstract}

\section{Hosted file}

Manuscript-PCE. doc available at https : //authorea.com/users/307777/articles/438743-elevated-ozonelevels-affect-metabolites-and-related-biosynthetic-genes-in-tartary-buckwheat

\section{Hosted file}

Figures 1-9-PCE.pptx available at https://authorea.com/users/307777/articles/438743-elevatedozone-levels-affect-metabolites-and-related-biosynthetic-genes-in-tartary-buckwheat 